\title{
Cation/anion co-doped and cobalt-free Li-rich layered cathode for high-performance Li-ion batteries
}

Lu Nie, Zeyu Wang, Xiaowen Zhao, Shaojie Chen, Yingjie He, Haojie Zhao, Tianyi

Gao, Yue Zhang, Lei Dong, Franklin Kim, Yi Yu, Wei Liu*

School of Physical Science and Technology, ShanghaiTech University, Shanghai,

201210, China

E-mail: liuwei1@shanghaitech.edu.cn 


\section{Experimental Methods}

Material Preparation: All four samples were synthesized via a simple sol-gel procedure. The detailed synthesis procedures were similar to our previous work ${ }^{1-2}$. For preparing samples, the corresponding stoichiometric of $\mathrm{Fe}\left(\mathrm{NO}_{3}\right)_{3} \cdot 9 \mathrm{H}_{2} \mathrm{O}(99.0 \%$, Sigma-Aldrich) and $\mathrm{NH}_{4} \mathrm{Cl}(99.0 \%$, Sigma-Aldrich) were added to the above mixed metal solution. Subsequent experiment steps were similar to the control sample.

Material Characterization: The morphology and size of the LLOs were observed with a JEOL JSM-7800F scanning electron microscopy (SEM) and an energy dispersive spectroscopy (EDS) was also used for further analysis. The microstruture of the samples was captured by a transmission electron microscope (TEM, FEI T12) operated at $200 \mathrm{kV}$ accelerating voltage. X-ray diffraction (XRD) tests were conducted on a Bruker D8 Advance and $\mathrm{Cu} \mathrm{K} \alpha$ radiation source in the two-theta range of $10-80^{\circ}$ at an interval of $0.02^{\circ}$. Total Pattern Solution (TOPAS) software was applied for the Rietveld refinement. For in-situ XRD measurement, a Microelab $\mathrm{X}-3-\mathrm{ION}-\mathrm{F}$ electrochemical cell equipped with $0.2 \mathrm{~mm}$ beryllium $(\mathrm{Be})$ window was used. X-ray photoelectron spectrum (XPS) tests were conducted on a Thermo Fisher ESCALAB $250 \mathrm{Xi}$. The Raman tests were performed on Renishaw inVia equipped with a $532 \mathrm{~nm}$ laser. Elemental molar fractions were revealed using inductively coupled plasma optical emission spectrometry (ICP-OES, Icap7400) analysis. Differential scanning calorimetry (DSC) analyses were performed on PerkinElmer DSC8000. 
Electrochemical Tests: The mixed electrode slurry containing $80 \mathrm{wt} \%$ active electrode materials, $10 \mathrm{wt} \%$ conductive carbon (Super $\mathrm{P}$ ), and $10 \mathrm{wt} \%$ polyvinylidene fluoride (PVDF) binder was coated on $\mathrm{Al}$ foil and then dried at $100{ }^{\circ} \mathrm{C}$ for $12 \mathrm{~h}$ under vacuum. Electrode disks $\left(1.13 \mathrm{~cm}^{2}\right.$ area) were punched with an average active material of $1-2 \mathrm{mg} \mathrm{cm}^{-2}$. The $60 \mu \mathrm{L}$ electrolyte solution of $1 \mathrm{M} \mathrm{LiPF}_{6}$ in ethylene carbonate (EC) / diethyl carbonate (DEC) (1:1 vol \%) was used. Electrochemical impedance spectra (EIS) tests were conducted on an electrochemical working station (VMP 300, Bio-Logic) at a frequency range of $100 \mathrm{kHz}$ to $100 \mathrm{mHz}$. Galvanostatic measurements were carried out using LAND battery test systems.

Theoretical calculation: The structure of $\mathrm{Li}_{1.2} \mathrm{Ni}_{0.185} \mathrm{Mn}_{0.585} \mathrm{Fe}_{0.03} \mathrm{O}_{1.98} \mathrm{Cl}_{0.02}$ is constructed to represent the structure of $\mathrm{Fe}, \mathrm{Cl}$ co-doped $\mathrm{Li}_{1.2} \mathrm{Ni}_{0.2} \mathrm{Mn}_{0.6} \mathrm{O}_{2}$ (ICSD: 154067) ${ }^{16}$. The $\mathrm{Li}_{1.2} \mathrm{Ni}_{0.2} \mathrm{Mn}_{0.6} \mathrm{O}_{1.98} \mathrm{Cl}_{0.02}$ structure is obtained by replacing the $\mathrm{Fe}$ atoms of $\mathrm{Li}_{1.2} \mathrm{Ni}_{0.185} \mathrm{Mn}_{0.585} \mathrm{Fe}_{0.03} \mathrm{O}_{1.98} \mathrm{Cl}_{0.02}$ with $\mathrm{Ni}$ atom and $\mathrm{Mn}$ atom. The Vienna ab initio simulation (VASP) package ${ }^{17}$ is applied in the first-principle calculations with the use of projector-augmented wave $(\mathrm{PAW})^{18}$ potentials. The generalized gradient approximation (GGA) with the Perdew-Burke-Ernzerhof (PBE) functional ${ }^{19}$ is used to treat the exchange-correlation functional. The plane wave energy cutoff is set to 500 $\mathrm{eV}$. A tetrahedron method with a $3 * 3 * 3 \mathrm{k}$-point mesh is used for the Brillouin zone. 
The binding energy $\mathrm{E}_{\mathrm{b}}$ is calculated by $\mathrm{E}_{\mathrm{b}}^{\mathrm{Fe}}=\mathrm{E}_{\mathrm{LNMOCl}-\mathrm{Ni}-\mathrm{Mn}}+2 \mathrm{E}_{\mathrm{Fe}}-\mathrm{E}_{\mathrm{LNMOFeCl}}$ and $E_{b}^{N i}+M n=E_{L N M O C l}-\mathrm{Ni}-M n+E_{N i}+E_{M n}-E_{L N M O C l}$, which describes the binding strength between them. Here, $\mathrm{E}_{\mathrm{LNMOCl}-\mathrm{Ni}-\mathrm{Mn}}$ represents the energy of $\mathrm{Li}_{1.2} \mathrm{Ni}_{0.185} \mathrm{Mn}_{0.585} \mathrm{VO}_{1.98} \mathrm{Cl}_{0.02}$ model, in which $\mathrm{V}$ represents cation vacancy, $\mathrm{E}_{\mathrm{LNMOFeCl}}$ is for the energy of $\mathrm{Li}_{1.2} \mathrm{Ni}_{0.185} \mathrm{Mn}_{0.585} \mathrm{Fe}_{0.03} \mathrm{O}_{1.98} \mathrm{Cl}_{0.02}$ model, $\mathrm{E}_{\mathrm{LNMOCl}}$ is for the energy of $\mathrm{Li}_{1.2} \mathrm{Ni}_{0.2} \mathrm{Mn}_{0.6} \mathrm{O}_{1.98} \mathrm{Cl}_{0.02}$ model, and $\mathrm{E}_{\mathrm{Fe}}, \mathrm{E}_{\mathrm{Ni}}, \mathrm{E}_{\mathrm{Mn}}$ are for the energy of each isolated atom.

The sample with 3 at $\%$ Fe-doping and 2 at $\%$ Cl-doping showed the optimal electrochemical performance. Fe can participate in the oxidation reaction of $\mathrm{Fe}^{3+}$ to $\mathrm{Fe}^{4+}$ to compensate part of the specific capacity loss. However, excessive Fe-doping will transform into the electrochemically inactive $\mathrm{LiFeO}_{2}$ phase and reduces specific capacity $^{3-4}$. Previous reports ${ }^{3,5}$ have shown that 3 at $\%$ Fe-doped material shows more excellent structural stability and electrochemical performance than 1 and 5 at $\%$ Fe-doped samples. Meanwhile, appropriate amount of Cl-doping can effectively improve the structural stability of the materials during the charging process ${ }^{6-7}$. However, on the one hand, the chlorine ions with larger doped concentration in the materials provide small electrons during the charging process, which will result in a more complex redox mechanism and the structural variation of the materials ${ }^{8}$. On the other hand, the average transition metal $\left(\mathrm{Mn}^{4+}\right)$ oxidation state tends to decrease for charge balance with the Cl-doping ${ }^{9}$, which is not conducive to maintaining structural stability and average voltage. 

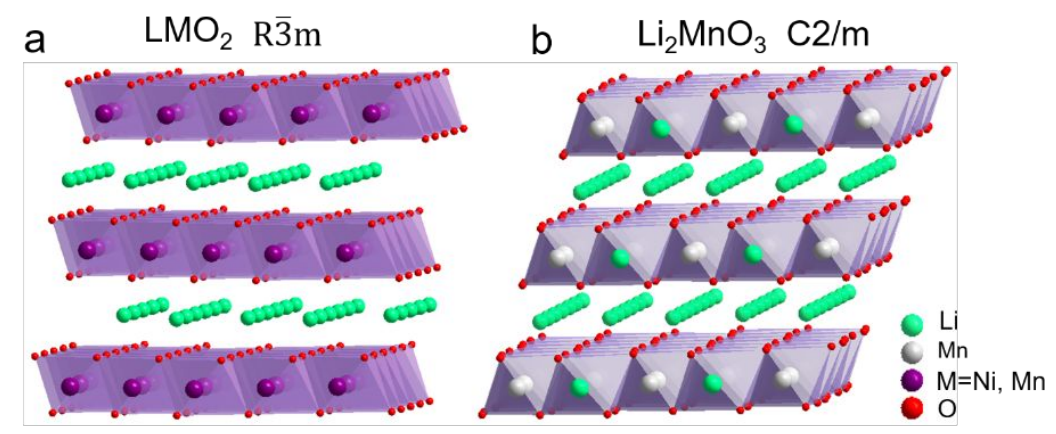

Figure S1. Structure of $\mathrm{LiMO}_{2}$ (a) and $\mathrm{Li}_{2} \mathrm{MnO}_{3}$ (b). The green, gray, purple, and red spheres represent $\mathrm{Li}$ atoms, $\mathrm{Mn}$ atoms, transition metal atoms, and $\mathrm{O}$ atoms, respectively.

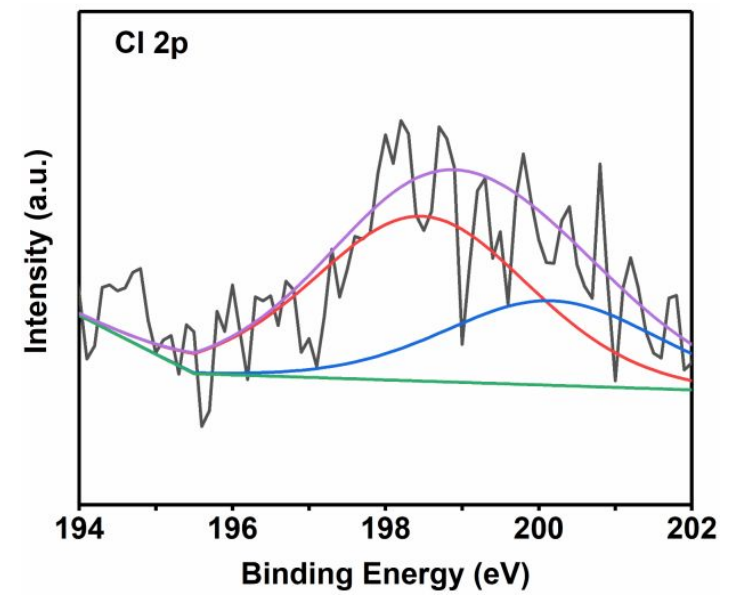

Figure S2. XPS Cl 2p spectrum of the Fe\&Cl-LNMO material. 


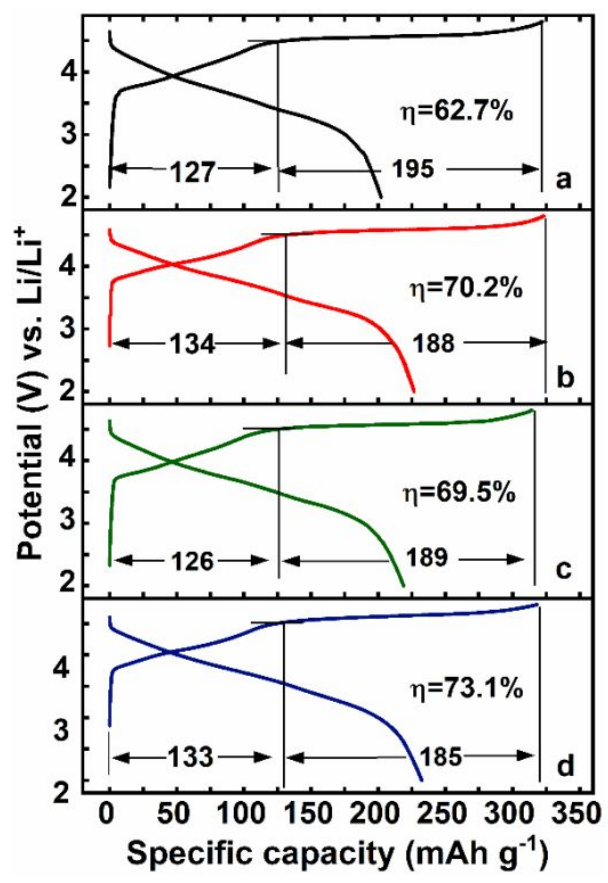

Figure S3 (a-d) Comparison of the initial voltage profiles of the pristine LNMO, Fe-LNMO, Cl-LNMO and Fe\&Cl-LNMO sample at a C-rate of $0.2 \mathrm{C}$.

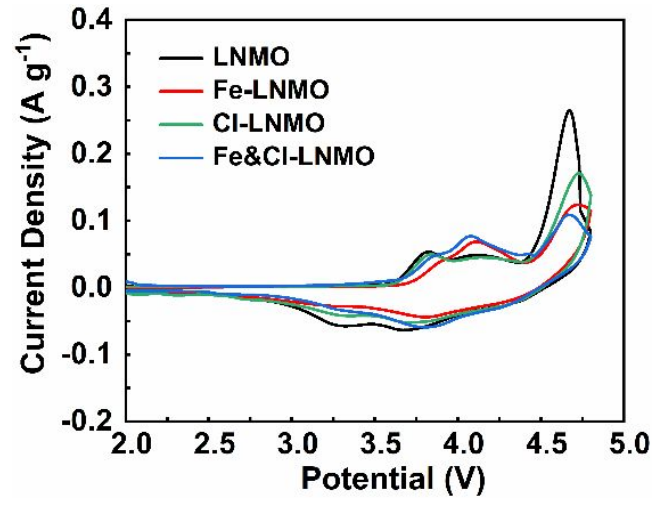

Figure S4. Cyclic voltammatry curves using a scan rate of $0.1 \mathrm{mV} \mathrm{s}^{-1}$ within the potential range from 2 to $4.8 \mathrm{~V} \mathrm{vs} \mathrm{Li}^{+} / \mathrm{Li}$. 


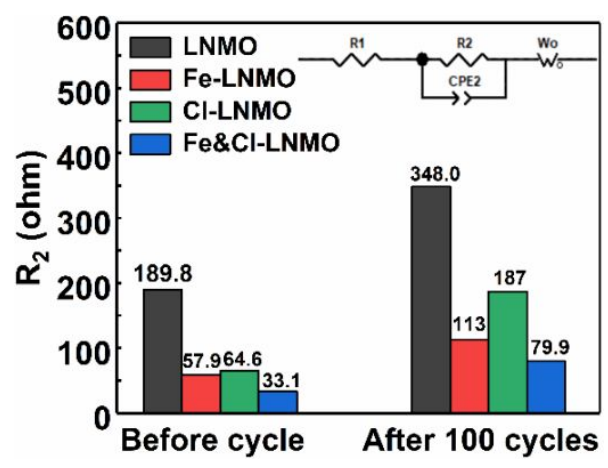

Figure S5. The interface resistance fitting results histogram before and after 100 cycles. The inset shows the equivalent circuit.

when the Li-rich materials are charged beyond $4.5 \mathrm{~V}$, the surface oxygen can be easily oxidized to oxygen and escapes from the material surface. The escaped oxygen can easily react with the electrolyte, continuously producing undesired side reaction products and increasing its interface impedance ${ }^{10-11}$. The calculations show that the covalent binding energy is enhanced by $\mathrm{Fe}$ and $\mathrm{Cl}$ co-doping with improved structural stability. In addition, the oxidation peak intensity at around $4.7 \mathrm{~V}$ related to the release of oxygen obviously decreases for the Fe\&Cl-LNMO sample compared with the undoped LNMO in the corresponding cyclic voltammogram profiles (Figure S4), indicating that the loss of $\mathrm{O}_{2}$ is well suppressed by the $\mathrm{Fe}$ and $\mathrm{Cl}$ doping with less side reactions and a more stable surface structure.
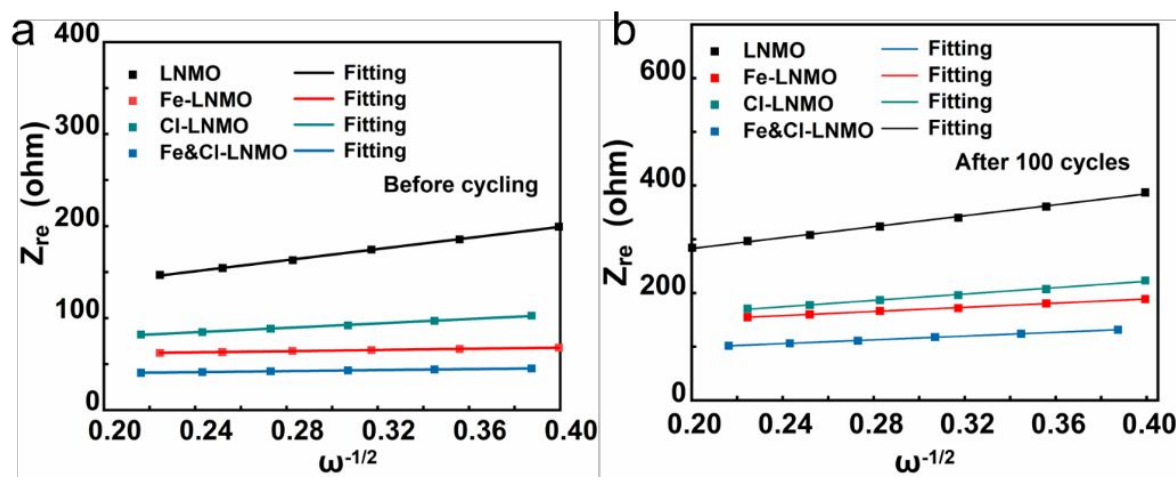

Figure S6. $\mathrm{Z}_{\mathrm{re}}-\omega^{-1 / 2}$ plots of the LNMO, Fe-LNMO, Cl-LNMO and Fe\&Cl-LNMO samples before and after 100 cycles.

In order to further verify the expansion of $\mathrm{Li}^{+}$diffusion channels, the change of $\mathrm{Li}$ slab space and TM slab space is calculated based on the formulas as follows ${ }^{12-14}$ :

$$
\begin{gathered}
S_{T M}=\left(\frac{2}{3}-2 Z_{O X}\right) \times C_{h e x} \\
I_{L i}=\frac{C_{\text {hex }}}{3}-S_{T M}
\end{gathered}
$$


where $\mathrm{S}_{\mathrm{TM}}$ and $\mathrm{I}_{\mathrm{Li}}$ represent the interslab spacing of transition metal layer and lithium layer, respectively. $Z_{o x}$ is taken to be $0.2411^{12,14-15}$, and $C_{h e x}$ is the lattice constant $c$. The lattice constant c of LNMO and Fe\&Cl-LNMO is $14.2219 \AA$ and $14.2479 \AA$, respectively. The interslab spacing of transition metal layer calculated by the formulas above are $2.6235 \AA$ and $2.6283 \AA$ for LNMO and Fe\&Cl-LNMO. The interslab spacing of lithium layer are $2.1171 \AA$ and $2.1210 \AA$ for LNMO and Fe\&Cl-LNMO. A larger Li slab space appears for Fe\&Cl-LNMO, which can effectively accelerate Li-ion diffusion and improve the rate performance of the materials.
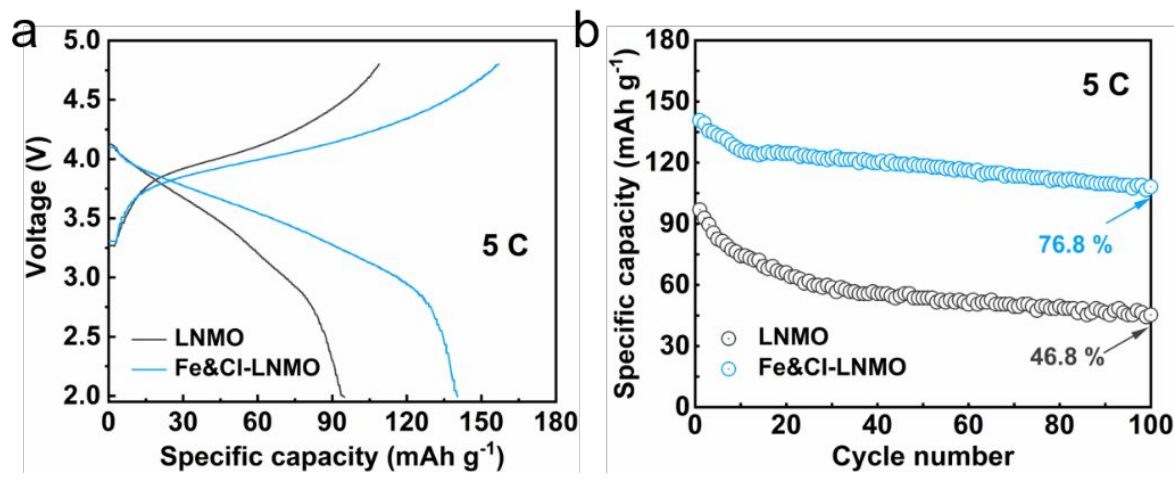

Figure S7. The charge/discharge curves and the cycling performance of the half cells using the LNMO and Fe\&Cl-LNMO samples at $5 \mathrm{C}$ and under room temperature. The half cells are initially activated at $0.2 \mathrm{C}$ for 1 cycle.

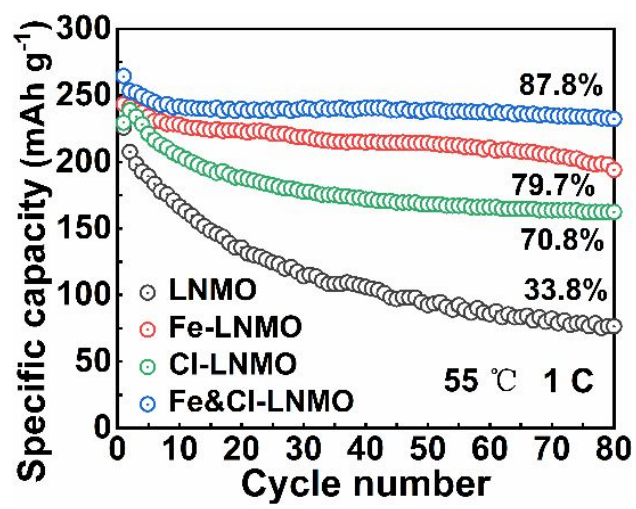

Figure S8. The cycling performance of the half cells at $55^{\circ} \mathrm{C}$ under $1 \mathrm{C}$. 


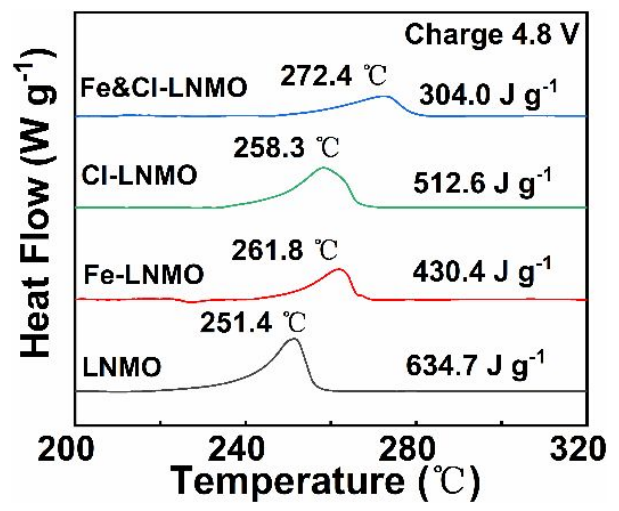

Figure S9. DSC profiles of the half-cells charged to $4.8 \mathrm{~V}$ at $0.2 \mathrm{C}$.

Table S1. Rietveld refinement results for the pristine LNMO, Fe-LNMO, Cl-LNMO and Fe\&Cl-LNMO sample.

\begin{tabular}{llllll}
\hline Sample & $\mathrm{a}(\AA)$ & $\mathrm{c}(\AA)$ & $\mathrm{c} / \mathrm{a}$ & $\mathrm{V}\left(\AA^{3}\right)$ & $\mathrm{I}_{003} / \mathrm{I}_{104}$ \\
\hline LNMO & $2.8549(1)$ & $14.2219(6)$ & 4.982 & $100.383(5)$ & $1.7925(0)$ \\
Fe-LNMO & $2.8569(2)$ & $14.2392(0)$ & 4.984 & $100.646(9)$ & $2.1408(5)$ \\
& & & & & \\
Cl-LNMO & $2.8559(5)$ & $14.2302(2)$ & 4.983 & $100.515(1)$ & $2.0496(1)$ \\
& & & & & \\
Fe\&Cl-LNMO & $2.8584(2)$ & $14.2479(9)$ & 4.985 & $100.814(8)$ & $2.4124(7)$ \\
\hline
\end{tabular}

Table S2. The exothermal temperature and heat release from DSC analysis using the pristine LNMO, Fe-LNMO, Cl-LNMO and Fe\&Cl-LNMO sample.

\begin{tabular}{lll}
\hline Sample & Exothermal temperature $\left({ }^{\circ} \mathrm{C}\right)$ & Heat release $\left(\mathrm{J} \mathrm{g}^{-1}\right)$ \\
\hline LNMO & 251.4 & 634.7 \\
\hline Fe-LNMO & 261.8 & 430.4 \\
\hline Cl-LNMO & 258.3 & 512.6 \\
\hline Fe\&Cl-LNMO & 272.4 & 304.0 \\
\hline
\end{tabular}




\section{Reference}

(1) Nie, L.; Li, Y.; Chen, S.; Li, K.; Huang, Y.; Zhu, Y.; Sun, Z.; Zhang, J.; He, Y.; Cui, M.; Wei, S.; Qiu, F.; Zhong, C.; Liu, W. Biofilm nanofiber-coated separators for dendrite-free lithium metal anode and ultrahigh-rate lithium batteries. ACS Appl. Mater. Interfaces 2019, 11, 32373-32380.

(2) Nie, L.; Liang, C.; Chen, S.; He, Y.; Liu, W.; Zhao, H.; Gao, T.; Sun, Z.; Hu, Q.; Zhang, Y.; Yu, Y.; Liu, W. Improved electrochemical performance of Li-rich layered oxide cathodes enabled by a two-step heat treatment. ACS Appl. Mater. Interfaces 2021, 13, 13281-13288.

(3) Wu, F.; Kim, G. T.; Kuenzel, M.; Zhang, H.; Asenbauer, J.; Geiger, D.; Kaiser, U.; Passerini, S. Elucidating the effect of iron doping on the electrochemical performance of cobalt - free lithium - rich layered cathode materials. Adv. Energy Mater. 2019, 9, 1902445.

(4) Tabuchi, M.; Nabeshima, Y.; Takeuchi, T.; Kageyama, H.; Tatsumi, K.; Akimoto, J.; Shibuya, H.; Imaizumi, J. Synthesis and electrochemical characterization of Fe and $\mathrm{Ni}$ substituted Li2MnO3 - an effective means to use Fe for constructing "Co-free" Li2MnO3 based positive electrode material. J. Power Sources 2011, 196, 3611-3622.

(5) Wang, C.-C.; Manthiram, A. Influence of cationic substitutions on the first charge and reversible capacities of lithium-rich layered oxide cathodes. J. Mater. Chem. A 2013, $1,10209$.

(6) Zhou, H.; Guan, H.; Yin, C.; Liu, B.; Li, J. A potassium/chloride ion co-doped cathode material Li1.18K0.02Ni0.2Mn0.6O1.98C10.02 with enhanced electrochemical performance for lithium ion batteries. J. Mater. Sci.: Mater. Electron. 2019, 31, 572-580.

(7) Zhu, H.; Li, Q.; Gong, X.; Cao, K.; Chen, Z. Enhanced high voltage performance of chlorine/bromine co-doped lithium nickel manganese cobalt oxide. Crystals 2018, $8,425$.

(8) Yan, H.; Li, B.; Yu, Z.; Chu, W.; Xia, D. First-principles study: tuning the redox behavior of lithium-rich layered oxides by chlorine doping. J. Phys. Chem. C 2017, 121, 7155-7163.

(9) Han, D.-W.; Ryu, W.-H.; Kim, W.-K.; Eom, J.-Y.; Kwon, H.-S. Effects of Li and $\mathrm{Cl}$ codoping on the electrochemical performance and structural stability of LiMn2O4 cathode materials for hybrid electric vehicle applications. J. Phys. Chem. C 2013, 117, 4913-4919.

(10) Sun, Z.; Xu, L.; Dong, C.; Zhang, H.; Zhang, M.; Ma, Y.; Liu, Y.; Li, Z.; Zhou, Y.; Han, Y.; Chen, Y. A facile gaseous sulfur treatment strategy for Li-rich and Ni-rich cathode materials with high cycling and rate performance. Nano Energy 2019, 63, 103887.

(11) Liu, D.; Fan, X.; Li, Z.; Liu, T.; Sun, M.; Qian, C.; Ling, M.; Liu, Y.; Liang, C. A cation/anion co-doped Li1.12Na0.08Ni0.2Mn0.6O1.95F0.05 cathode for lithium ion batteries. Nano Energy 2019, 58, 786-796.

(12) Zhong, J.; Yang, Z.; Yu, Y.; Liu, Y.; Li, J.; Kang, F. Surface substitution of polyanion to improve structure stability and electrochemical properties of lithium-rich 
layered cathode oxides. Appl. Surf. Sci 2020, 512, 145741.

(13) Xu, G.; Xue, Q.; Li, J.; Li, Z.; Li, X.; Yu, T.; Li, J.; Wang, X.; Kang, F. Understanding the enhanced electrochemical performance of samarium substituted $\mathrm{Li}[\mathrm{Li} 0.2 \mathrm{Mn} 0.54-\mathrm{xSm} x \mathrm{Co} 0.13 \mathrm{Ni} 0.13] \mathrm{O} 2$ cathode material for lithium ion batteries. Solid State Ionics 2016, 293, 7-12.

(14) Song, B.; Lai, M. O.; Lu, L. Influence of $\mathrm{Ru}$ substitution on Li-rich $0.55 \mathrm{Li} 2 \mathrm{MnO} 3 \cdot 0.45 \mathrm{LiNi} 1 / 3 \mathrm{Co} 1 / 3 \mathrm{Mn} 1 / 3 \mathrm{O} 2$ cathode for Li-ion batteries. Electrochim. Acta 2012, 80, 187-195.

(15) Li, L.; Song, B. H.; Chang, Y. L.; Xia, H.; Yang, J. R.; Lee, K. S.; Lu, L. Retarded phase transition by fluorine doping in Li-rich layered Li $1.2 \mathrm{Mn} 0.54 \mathrm{Ni}$ 0.13 Co 0.13 O 2 cathode material. J. Power Sources 2015, 283, 162-170.

(16) Yasushi Idemoto, Hiroshi Sekine, Koichi Ui, Nobuyuki Koura. Crystal structural change during charge-discharge process of LiMn1.5Ni0.5O4 as cathode material for $5 \mathrm{~V}$ class lithium secondary battery. Solid State Ionics 2005, 176.

(17) Institut für Theoretische Physik, Technische Universita“t Wien, Wiedner Hauptstrabe. Efficient iterative schemes for ab initio total-energy calculations using a plane-wave basis set. PHYSICAL REVIEW B. 1996, 54.

(18) Peter E. Blöchl, O. Jepsen, and O. K. Andersen. Improved tetrahedron method far Brilleuin-zane integratians. PHYSICAL REVIEW B 1994, 49.

(19) John P. Perdew, Kieron Burke, Matthias Ernzerhof. Generalized gradient approximation made simple. PHYSICAL REVIEW LETTERS 1996, 77. 